\title{
Control in a Dissipative Environment: The Example of a Cope Rearrangement
}

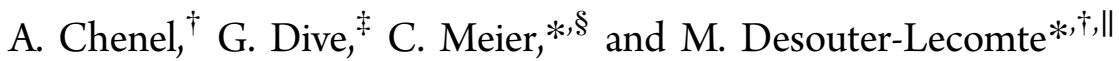 \\ ${ }^{\dagger}$ Laboratoire de Chimie Physique, Université Paris-Sud and CNRS, UMR 8000, F-91405 Orsay, France \\ ‡Centre d’Ingénierie des Protéines, Université de Liège, Sart Tilman, B6, B-4000 Liège, Belgium \\ ${ }^{\S}$ LCAR-IRSAMC, Université Paul Sabatier, 31062 Toulouse, France \\ "Département de Chimie, Université de Liège, Sart Tilman, B6, B-4000 Liège, Belgium
}

ABSTRACT: In this work, we present optimal control calculations in a dissipative environment. To this end, the auxiliary density matrix method describing the dissipative quantum dynamics is combined with optimal control theory. The resulting approach, which is nonperturbative in the lasersystem interaction, is applied to model the control of Cope's isomerization of the methyl-cyclopentadienylcarboxylate dimer, described as the motion along a one-dimensional reaction path. The construction of the reaction path model as well as the dipole moments required for the laser interaction

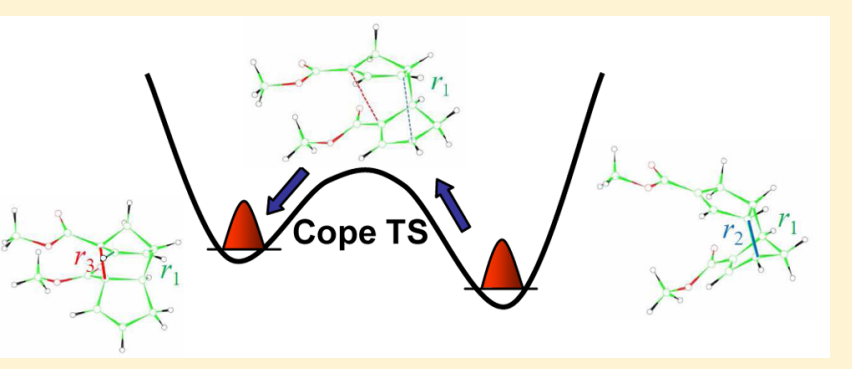
are obtained from DFT quantum chemistry calculations. As a main result, we show that the proposed methodology, which includes the environment at the design stage of the control, leads to control fields which can react on dissipative effects during the dynamics and lead to an increased control objective, as compared to control fields obtained without dissipation. The chosen example is analyzed in detail, and the physical mechanisms of the control under dissipation are elucidated.

\section{INTRODUCTION}

Coherent control of molecular systems has been the focus of numerous theoretical and experimental investigations starting from the pico- or femtosecond domain ${ }^{1-9}$ to reach nowadays the attosecond range and electronic control. ${ }^{10-13}$ Control of isomerization reactions by designed laser pulses has been investigated since the early days of laser chemistry. ${ }^{14-30}$ Isomerization involves population transfer from a potential well to another one, and different control strategies have been suggested either in the UV domain via the electronic excited states in the pump and dump scheme ${ }^{18-23,31}$ or in the infrared range in the ground electronic state by overcoming the barrier via the delocalized highly excited vibrational states. $^{14-17,24-30,32-34}$ We focus here on the control of nuclear motion during an isomerization in the ground electronic state. Feedback loop control which is an efficient experimental technique to modify reactivity both in isolated conditions and in the condensed phase has been reviewed recently. ${ }^{35}$ As discussed in this paper, ${ }^{35}$ numerical simulations in complex systems are often oversimplified to be predictive in laser design due to the very sensitive response of a quantum system to external fields and the limited precision that any numerical simulation in complex systems has to face. However, simulations are important to explore the feasibility of control in different systems, and to analyze the mechanisms behind successful control fields. Furthermore, the control on nonisolated systems presents an additional challenge, which stems from the influence of the environment. Indeed, quantum control by optimally shaped laser pulses exploits fine quantum interferences in the system and is therefore extremely sensitive to decoherence due to the uncontrolled surrounding. Simulations in open quantum systems have been performed by commonly used approximations as weak system-surrounding coupling with a time local Markovian approach in the Redfield ${ }^{15,16,19,36-39}$ or Lindblad formalism. ${ }^{40-43}$ More sophisticated non-Markovian methods, more accurate to include driving-dissipation correlation, have also been explored. ${ }^{14,44-46}$

Here, we focus on a particular isomerization in the framework of the Diels-Alder reaction inspired by Manz' theoretical investigation of a Cope rearrangement in substituted semibullvalenes. ${ }^{14}$ This pioneering work was one of the first models of a control by a non-Markovian approach. In a recent paper, the dimerization of methyl-cyclopentadienylcarboxylate has been explored experimentally and theoretically using quantum chemistry methods. ${ }^{47}$ The dimerization of methylcyclopentadienylcarboxylate can involve different isomers, but the major product is known to be Thiele's ester. ${ }^{48,49}$ This ester is the reactant for the control, and the target is the product of the Cope rearrangement. A one-dimensional reaction path model has been developed, which forms the basis of the isomerization control presented in this work. The effect of the surrounding is modeled by coupling the reaction path

Special Issue: Jörn Manz Festschrift

Received: May 30, 2012

Revised: August 21, 2012

Published: August 22, 2012 
coordinate to a bath of harmonic oscillators. At this stage, different dynamical strategies could be followed: an extensive quantum computation with the multilayer multiconfiguration time dependent Hartree (MCTDH) method up to some hundreds of degrees of freedom ${ }^{50}$ or dissipative dynamics in which the surrounding is taken into account by a global spectral density. ${ }^{51}$ Here, we implement a non-Markovian dissipative dynamics in the density matrix formalism which is second order in the system-bath coupling but with no limitation on the strength of the field. ${ }^{52}$ Such a time nonlocal non-Markovian approach with a memory including the whole dynamics from the initial time allows the system and the bath to have similar dynamical time scales. Following the particular Meier-Tannor parametrization of the spectral density of the bath, ${ }^{52-54}$ the field-dressed dissipation is treated by a set of auxiliary density matrices implicitly containing the memory terms and coupled to the system. This leads to time local dynamics which nevertheless takes finite memory and the effect of strong driving fields onto the dissipation fully into account. This specific non-Markovian master equation is here combined with optimal control theory (OCT). A similar approach going beyond second-order perturbation in the bath-system coupling has also been proposed ${ }^{45}$ but applied to small models.

The purpose of this work is to theoretically develop this formalism, to present its numerical implementation, and to show the effects of an environment on a controlled realistic isomerization reaction using the example mentioned above. To fully assess the role of dissipation, we first use a control field obtained without dissipation in a dynamics simulation including dissipation, and then compare these results to those obtained with a control field optimized with the presented formalism, which includes the dissipation already at the design stage.

As a main result, we show that our method that includes the bath leads to higher target yields, and the mechanism the control field adopts in order to avoid or minimize bath-induced decoherence is analyzed.

The paper is organized as follows: In the next section, we give details on the system under study, its characterization, and the construction of the reaction path Hamiltonian, based on electronic structure theory. The following chapter is devoted to the dynamical model and the treatment of the model bath through a non-Markovian master equation. Then, the optimal control theory in the context of dissipative quantum evolution is presented, and combined with the master equation approach developed in the chapter before. That methodology is subsequently applied to the isomerization reaction of the dimer of methyl-cyclopentadienylcarboxylate, the results of which are presented in the Results section. The paper concludes with general remarks and an outlook to possible future directions in the context of the presented results.

\section{ELECTRONIC STRUCTURE CALCULATION OF THE REACTION PATH}

The dynamical studies are all based on potential energy curves and dipole moments calculated by density functional theory (DFT). Specifically, all electronic structure calculations were performed with the B3LYP functional, ${ }^{55}$ as implemented in the Gaussian program suite, ${ }^{56}$ using the double- $\zeta$ basis set 6$31 \mathrm{G}(\mathrm{d}) .{ }^{57}$

As stated in the Introduction, we are interested in the Cope rearrangement of the dimer of methyl-cyclopentadienylcarboxylate. The dimerization reaction leads primordially to Thiele's ester (structure a in Figure 1), which can isomerize via the
Cope transition state (structure b in Figure 1) to another stable form of higher energy, denoted $c$ in Figure 1.

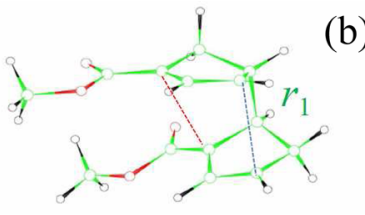

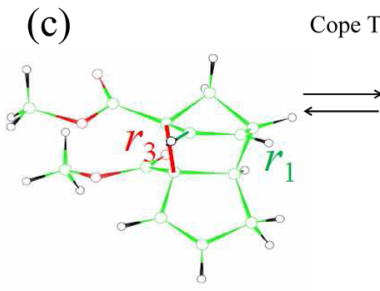

Target state (b)

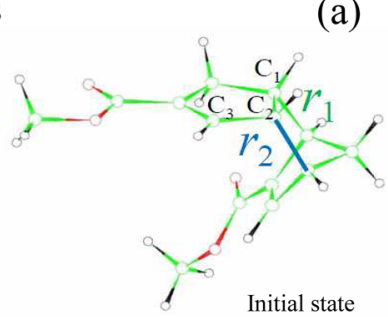

Figure 1. Geometries of Thiele's ester (a), the Cope transition state (b), and the product of the Cope rearrangement (c). Structures a and $\mathrm{c}$ are, respectively, the initial and target states for the laser control. The $\mu_{z}$ component of the dipole moment is along the $\mathrm{C}_{1}-\mathrm{C}_{2}$ bond, $\mu_{x}$ is perpendicular to the $\mathrm{C}_{1}-\mathrm{C}_{2}$ axis in the plane $\mathrm{C}_{1}-\mathrm{C}_{2}-\mathrm{C}_{3}$, and $\mu_{y}$ is perpendicular to this $\mathrm{C}_{1}-\mathrm{C}_{2}-\mathrm{C}_{3}$ plane. The dipole moments $\mu_{x}$ and $\mu_{y}$ chosen for the control are shown in Figure 2.

The main features of the rearrangement can be understood in terms of two main internuclear distances connecting the two cycles. They are denoted by $r_{2}$ and $r_{3}$ (see Figure 1 ). In the TS structure, the cycles are linked by a single strong bond denoted $r_{1}$ with an internuclear distance $r_{1}=1.6 \AA$. The two other bonds which can achieve the dimerization toward a stable isomer are drawn in dotted lines. In the most stable configuration of the dimer, Thiele's ester, the $r_{2}$ bond is formed with an internuclear distance of $1.6 \AA$. This structure is taken to be the reactant in our study.

The isomerization reaction proceeds by breaking this $r_{2}$ bond and forming a new bond $r_{3}$ with a distance of $1.6 \AA$. The energy and the values of the three important coordinates $r_{1}, r_{2}$, and $r_{3}$ are given in Table 1 . The three equilibrium structures of the Cope TS and the two adducts are depicted in Figure 1.

Table 1. Energy (in eV) and Geometrical Properties (in Å) of the Isomerization Reaction (See Figure 1)

\begin{tabular}{lcccc}
\multicolumn{1}{c}{ structure } & energy & $r_{1}$ & $r_{2}$ & $r_{3}$ \\
Thiele's ester (a) & 0 & 1.6 & 1.6 & 3.5 \\
Cope transition state (b) & 1.16 & 1.6 & 2.7 & 2.8 \\
structure (c) & 0.45 & 1.6 & 3.4 & 1.6 \\
\hline
\end{tabular}

Starting from the Cope TS structure, the IRC has been searched along the forward and reverse directions in massweighted Cartesian coordinates. The reverse branch could lead to the most stable minimum but stops after six steps. Using the standard Z-matrix coordinates, the optimization leads to the vicinity of the minimum but stops at an $r_{2}$ distance of $1.69 \AA$, while the adduct distance at equilibrium is $1.57 \AA$ and is $6.0 \mathrm{kcal}$ more stable than this last IRC point. Also in Cartesian coordinates or using a Z-matrix description, the forward branch comes back and does not go in the desired direction. In the surrounding of the TS, the surface is particularly flat. To overcome this problem, another starting point has been arbitrarily chosen, setting the $r_{3}$ distance to $2.6 \AA$. At that 
nonequilibrium structure, the Hessian matrix has a negative eigenvalue and the associated eigenvector is mainly defined by $r_{3}$. Now, the IRC starts in the right direction and stops near the second minimum with $r_{2}=3.43 \AA$ and $r_{3}=1.63 \AA$, to be compared to the optimized structure values which are 3.45 and $1.63 \AA$, respectively.

The energy profile along the reaction path has been approximated by a minimum energy profile scanned along $r_{2}$ and $r_{3}$ by steps of $0.5 \AA$ starting from the TS structure in Zmatrix description without any other constraints up to $1.45 \AA$ for both bonds. The good agreement between the three bond lengths obtained by these scans and the main IRC points can be noted. This minimum energy profile as a function of the coordinate $r=r_{3}-r_{2}$ forms the potential energy curve for the quantum dynamical studies. The system interaction with the external fields is based on the dipole moments $\mu_{x}$ and $\mu_{y}$, where the $x$-direction is perpendicular to the $\mathrm{C}_{1}-\mathrm{C}_{2}$ bond in the $\mathrm{C}_{1}-$ $\mathrm{C}_{2}-\mathrm{C}_{3}$ plane and the $y$-direction is perpendicular to that plane (see Figure 1). These two polarizations are preferred because they provide successive regions with a strong variation along the reaction path. The derivative of $\mu_{x}$ is large in the two wells, while $\mu_{y}$ is quickly varying in the TS region where $\mu_{x}$ is near an extremum. On the contrary, $\mu_{z}$ remains quasi-constant in the reactive well.

The resulting potential and dipole moments are depicted in Figure 2.
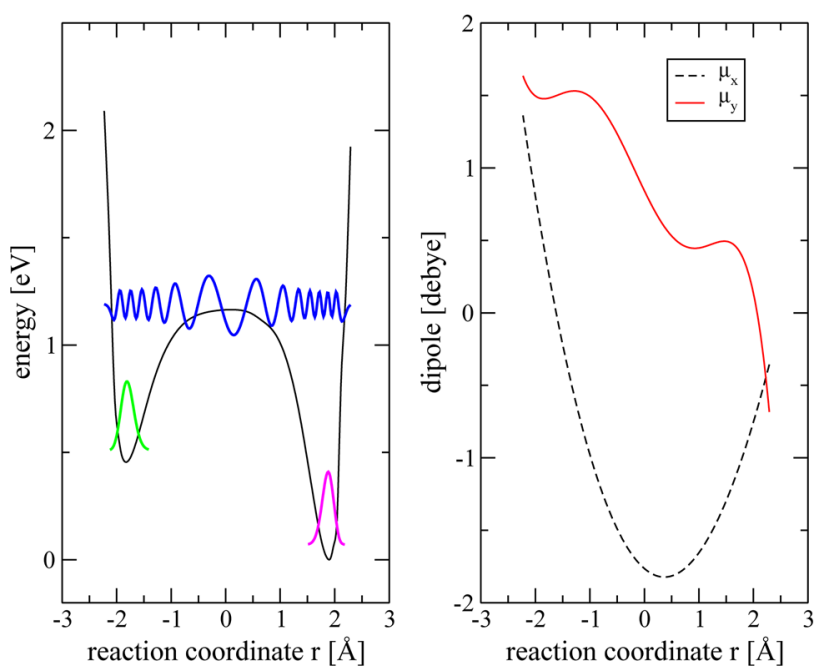

Figure 2. Potential energy curve along the reaction path parametrized by $r=r_{3}-r_{2}$ and Cartesian components of the dipole moment used in the control. The axes are defined in Figure 1. $\phi_{\nu=0}, \phi_{\nu=4}$, and $\phi_{\nu=26}$ are also drawn in the left panel (see the Results section).

\section{DYNAMIC MODEL AND NON-MARKOVIAN MASTER EQUATION}

System-Bath Model. We consider a one-dimensional model with the reaction path coordinate given by $r=r_{3}-r_{2}$. The potential energy curve along this path $V(r)$ is shown in Figure 2. In the $r_{2}, r_{3}$ subspace, we adopt a Cartesian kinetic energy operator with no cross term, which is justified since both coordinates have no common atom. The corresponding masses are $\mu_{2}=\mu_{3}=\mu_{\mathrm{C}} / 2$, with $\mu_{\mathrm{C}}$ being the mass of a $\mathrm{C}$ atom. By taking the sum and difference $R=r_{3}+r_{2}$ and $r=r_{3}-r_{2}$, the kinetic energy remains separable and the kinetic energy of the reaction path coordinate $r$ is given by $T_{r}=\left(\hbar^{2} / 2 \mu_{r}\right)\left(\partial^{2} / \partial r^{2}\right)$, with $\mu_{r}=\mu_{\mathrm{C}} / 4$. Hence, the Hamiltonian describing the motion along the reaction path, coupled to a bath of harmonic oscillators and interacting with external fields can be written as

$$
H(t)=H_{\mathrm{S}}(t)+H_{\mathrm{B}}+H_{\mathrm{SB}}+H_{\text {ren }}
$$

with $H_{S}(t)=T_{r}+V(r)-\sum_{j} \mu_{j}(r) E_{j}(t)$, where the last term describes the light-matter interaction at the electric dipolar approximation and $j$ denotes the chosen linear polarizations of the field. In this expression, $H_{\mathrm{B}}=\sum_{i=1}^{N} T_{i}+m_{i} \omega_{i}^{2} q_{i}^{2} / 2$ is a collection of harmonic oscillators which mimic the effects of the remaining modes and the solvent environment. The coupling of the reaction coordinate $r$ to the bath of harmonic oscillators is taken to be bilinear according to $H_{\mathrm{SB}}=-\sum_{i=1}^{N} c_{i} q_{i} r=-Q r$, where $Q=\sum_{i=1}^{N} c_{i} q_{i}$ is a collective bath coordinate. This leads to a renormalization term $H_{\text {ren }}=K r^{2} / 2$, with $K=\sum_{i=1}^{N} c_{i}^{2} / m_{i} \omega_{i}^{2}$. For later convenience, we also define effective system Hamiltonians, involving only the system coordinate, by $H_{\text {eff }}^{0}=$ $T_{r}+V(r)+H_{\text {ren }}$ and $H_{\text {eff }}=H_{\text {eff }}^{0}-\sum_{j} \mu_{j}(r) E_{j}(t)$. The coupling to the environment is determined by the coupling coefficients $c_{i}$, the distribution of which is given by the spectral density defined as

$$
J(\omega)=\frac{\pi}{2} \sum_{i=1}^{N} \frac{c_{i}^{2}}{m_{i} \omega_{i}} \delta\left(\omega-\omega_{i}\right)
$$

The bath is chosen to be Ohmic; i.e., we assume $J(\omega)=$ $\lambda \omega \mathrm{e}^{-\omega / \omega_{c}}$, with the parameter $\lambda$ which scales the system-bath coupling and $\omega_{c}$ which is a high-frequency cutoff. This type of bath has been widely used in previous studies on dissipative dynamics. ${ }^{58}$ This is a very general system-bath Hamiltonian, which has been considered in the field of dissipative quantum systems for a long time. On the basis of the seminal work of Caldeira and Leggett, ${ }^{59}$ it has been studied in a wide range of areas, like quantum optics, chemical physics, or solid state physics. $^{60}$

In our context, the model can be rationalized by considering that the motion along a reaction path induces changes in the large number of remaining modes. As a consequence, the primary isomerization motion is coupled to the other modes of the molecule and the solvent. In the case where the changes in the remaining modes are small, a microscopic model can be established, and the coupling strength is related to the reorganization energy of each coupled mode. ${ }^{61}$ However, in complex environments, the bath spectral density can be very complicated, and might not be well described by an Ohmic bath with cutoff (see the recent, very detailed work presented in ref 50). In the work presented here, we use the Ohmic bath as a model environment, and do not attempt to construct an underlying microscopic model. The main reason is that the Cope TS is very flat, and models based on a harmonic approximation of the potential energy surface around the TS seem to be questionable.

The model bath is described by two parameters, the coupling strength and the high-frequency cutoff. The latter can be understood as the highest frequency in a molecular system representing a vibrational motion which is coupled to the reaction path. Using $\omega_{\mathrm{c}}=1700 \mathrm{~cm}^{-1}$ leads to a bath of main frequencies in the range $1000-2000 \mathrm{~cm}^{-1}$, which represents the main vibrational motion of the molecular system. The coupling strength was used as a free parameter, and varied up to a value of $\lambda=0.008 \mathrm{au}$. This corresponds to a weak coupling regime, as can be estimated from the ratio $\gamma=\left(\lambda / \mu_{\mathrm{r}}\right) / \omega^{\#} \approx 0.004\left(\omega^{\#} \approx\right.$ 
$80 \mathrm{~cm}^{-1}$ is the imaginary frequency of the reaction path at the TS). ${ }^{62,63}$

Within a second-order perturbative approach with respect to the system-bath coupling, the entire bath dynamics enters into the system dynamics via the correlation function of the collective coordinate $C(t)=\langle Q(t), Q(0)\rangle_{\mathrm{B}}$, where \langle\rangle$_{\mathrm{B}}$ denotes an average over the bath at equilibrium. $C(t)$ is related to the spectral density of the bath by

$$
\begin{aligned}
C(t)= & \frac{1}{2 \pi} \int_{-\infty}^{\infty} \mathrm{d} \omega J(\omega)[\cos (\omega t) \operatorname{coth}(\beta \omega / 2) \\
& -i \sin (\omega t)]
\end{aligned}
$$

where the temperature enters via $\beta=1 / k_{\mathrm{B}} T$.

The value of $\omega_{\mathrm{c}}=1700 \mathrm{~cm}^{-1}$ chosen in this work yields a correlation function which decays in about $5 \mathrm{fs}$. This value, which reflects the bath memory, is short compared to the laser pulses and the typical system dynamics, which means that a Markovian approximation might be appropriate.

Note, however, that the methodology presented in the next section is a non-Markovian approach; i.e., no assumption of the bath correlation function is made, and is thus valid for any cutoff chosen.

Auxiliary Density Matrix Method. Open systems are generally described within the density matrix formalism. ${ }^{44,45,51-54,60}$ The reduced density matrix $\rho_{\mathrm{S}}(t)$ associated with the system is obtained by tracing over the bath coordinates. In the Nakajima-Zwanzig formalism, ${ }^{60} \rho_{\mathrm{S}}(t)$ is the solution of a reduced equation containing a memory which depends on the whole history of the global system-bath. Assuming factorizing initial conditions, the time evolution of $\rho_{\mathrm{S}}$ is given by

$$
\dot{\rho}_{\mathrm{S}}(t)=L_{\text {eff }} \rho_{\mathrm{S}}(t)+\int_{0}^{t} \mathrm{~d} t^{\prime} K\left(t, t^{\prime}\right) \rho_{\mathrm{S}}\left(t^{\prime}\right)
$$

with the memory kernel

$$
\begin{aligned}
K\left(t, t^{\prime}\right) \rho_{\mathrm{S}}\left(t^{\prime}\right)= & -\left[r, C\left(t-t^{\prime}\right) T \mathrm{e}^{\int_{t^{\prime}}^{t} L_{\mathrm{S}}\left(t^{\prime \prime}\right) \mathrm{d} t^{\prime \prime}} r \rho_{\mathrm{S}}\left(t^{\prime}\right)\right] \\
& +\left[r, C^{*}\left(t-t^{\prime}\right) T \mathrm{e}^{\int_{t^{\prime}}^{t} L_{\mathrm{S}}\left(t^{\prime \prime}\right) \mathrm{d} t^{\prime \prime}} \rho_{\mathrm{S}}\left(t^{\prime}\right) r\right]
\end{aligned}
$$

In these expressions, the Liouville operator is defined by $L_{\text {eff }} \bullet=$ $-i\left[H_{\text {eff }} \bullet\right]$ (atomic units are used throughout) and $T$ denotes the time ordering operator. The basic idea of the auxiliary matrix method is to use a fit of any given spectral density ${ }^{52}$ as a sum of $m$ Lorentzian functions

$$
J(\omega)=\frac{\pi}{2} \sum_{l=1}^{m} p_{1} \frac{\omega}{\left[\left(\omega+\Omega_{l}\right)^{2}+\Gamma_{l}^{2}\right]\left[\left(\omega-\Omega_{l}\right)^{2}+\Gamma_{l}^{2}\right]}
$$

so that $C(t)$ can be expressed as a sum over the poles in the complex plane. Note that, apart from the poles that stem from $J(\omega)$, an infinite number of poles for a nonvanishing temperature comes from the hyperbolic cotangent function in $C\left(t-t^{\prime}\right)$. Hence, we can write

$$
C\left(t-t^{\prime}\right)=\sum_{k=1}^{n} \alpha_{k} \mathrm{e}^{i \gamma_{k}\left(t-t^{\prime}\right)}
$$

$$
\begin{gathered}
\alpha_{2 l-1}=\frac{p_{l}}{8 \Omega_{l} \Gamma_{l}}\left[\operatorname{coth}\left(\beta\left(\Omega_{l}+i \Gamma_{l}\right) / 2\right)-1\right] \\
\alpha_{2 l}=\frac{p_{l}}{8 \Omega_{l} \Gamma_{l}}\left[\operatorname{coth}\left(\beta\left(\Omega_{l}-i \Gamma_{l}\right) / 2\right)+1\right] \\
\gamma_{2 l-1}=\Omega_{l}+i \Gamma_{l} \\
\gamma_{2 l}=-\Omega_{l}+i \Gamma_{l}
\end{gathered}
$$

for $l=1, \ldots, m$, generating $2 m$ terms that stem from $J(\omega)$ and

$$
\begin{aligned}
& \alpha_{k}=2 i J\left(i \nu_{k}\right) / \beta \\
& \gamma_{k}=i \nu_{k}
\end{aligned}
$$

for $k>2 m$, where $\nu_{k}=2 \pi(k-2 m) / \beta$ are the Matsubara frequencies which stem from the coth function. In practice, the number of Matsubara frequencies is truncated at a sufficiently large value.

Different strategies exist to express the complex conjugate $C^{*}\left(t-t^{\prime}\right)$ of the correlation function. Apart from straightforward conjugation of each term, one can also express $C^{*}\left(t-t^{\prime}\right)$ as

$$
C^{*}\left(t-t^{\prime}\right)=\sum_{k=1}^{n} \tilde{\alpha}_{k} \mathrm{e}^{i \gamma_{k}\left(t-t^{\prime}\right)}
$$

with the same exponents as in eq 7 but different coefficients $\tilde{\alpha}_{k}$. Specifically, one finds $\tilde{\alpha}_{2 l-1}=\alpha_{2 l}^{*}, \tilde{\alpha}_{2 l-1}=\alpha_{2 l-1}^{*}$ for $l=1, \ldots, m$ and $\tilde{\alpha}_{k}=\alpha_{k}$ for $k>2 m$. Note that this way of writing $C^{*}\left(t-t^{\prime}\right)$ involves the same exponents as in $C\left(t-t^{\prime}\right)$, which is a consequence of the symmetries inherent in eq 3 , and which leads to a compact form to treat the memory kernel given in eq 5. A detailed derivation of these relations is given in ref 54. By inserting these expressions into eq 4, the integral of the memory term is split into a sum of contributions for each component $k$ of the correlation function and each partial integral is set equal to an auxiliary matrix

$$
\rho_{k}(t)=i \int_{0}^{t} \mathrm{~d} t^{\prime} \mathrm{e}^{i \gamma_{k}\left(t-t^{\prime}\right)} T \mathrm{e}^{\int_{t^{\prime}}^{t} L_{\mathrm{S}}\left(t^{\prime \prime}\right) \mathrm{d} t^{\prime \prime}}\left(\alpha_{k} r \rho_{\mathrm{S}}\left(t^{\prime}\right)-\tilde{\alpha}_{k} \rho_{\mathrm{S}}\left(t^{\prime}\right) r\right)
$$

By taking the time derivative of this integral, one obtains a set of coupled equations for the density matrix of the system $\rho_{\mathrm{S}}(t)$ and the $n$ auxiliary matrices $\rho_{k}(t)$. The main point is that this technique allows us to carry out non-Markovian dynamics by a system of coupled equations local in time.

The equations take the form

$$
\begin{aligned}
& \dot{\rho}_{\mathrm{S}}(t)=L_{\mathrm{eff}} \rho_{\mathrm{S}}(t)+i \sum_{k}\left[r, \rho_{k}(t)\right] \\
& \dot{\rho}_{k}(t)=\left(i \gamma_{k}+L_{\mathrm{S}}\right) \rho_{k}(t)+i\left[\alpha_{k} r \rho_{\mathrm{S}}(t)-\tilde{\alpha}_{k} \rho_{\mathrm{S}}(t) r\right]
\end{aligned}
$$

The coupled equations have been integrated by the splitoperator technique. ${ }^{64}$ In matrix form, the system reads

$$
\left(\begin{array}{c}
\dot{\rho}_{\mathrm{S}}(t) \\
\dot{\rho}_{1}(t) \\
\vdots \\
\dot{\rho}_{k}(t) \\
\vdots
\end{array}\right)=\left(\begin{array}{cccccc}
L_{\text {eff }}(t) & L^{-} & & \ldots & & L^{-} \\
O_{1} & L_{\mathrm{S}}+i \gamma_{1} & & & & \\
\vdots & & \ddots & & & \\
O_{k} & & & L_{\mathrm{S}}(t)+i \gamma_{k} & \\
\vdots & & & & \ddots &
\end{array}\right)\left(\begin{array}{c}
\rho_{\mathrm{S}}(t) \\
\rho_{1}(t) \\
\vdots \\
\rho_{k}(t) \\
\vdots
\end{array}\right)
$$


where $L_{S} \bullet=-i\left[H_{S}, \bullet\right], O_{k}=1 / 2\left[\alpha_{k}\left(L^{-}+L^{+}\right)+\tilde{\alpha}_{k}\left(L^{-}-L^{+}\right)\right]$ with $L^{-} \bullet=i[r, \bullet]$ (i.e., a commutator) and $L^{+}=i[r, \bullet]_{+}$(i.e., an anticommutator). In a more concise form, this can be written as

$$
\partial_{t} \hat{\rho}(t)=\left(L_{\text {diag }}+L_{\text {off }}\right) \hat{\rho}(t)
$$

where $\hat{\rho}(t)$ is a vector containing the system $\rho_{\mathrm{S}}(t)$ and auxiliary density matrices $\rho_{k}(t)$ and $L_{\text {diag }}$ and $L_{\text {off }}$ are the diagonal and offdiagonal matrix blocks of eq 14 containing the operators in Liouville space. By splitting the diagonal and off-diagonal part, one gets $\hat{\rho}(t+\delta t)=e^{L_{\text {off }} \delta t / 2} e^{L_{\text {diag }} \delta t} e^{L_{\text {off }} \delta t / 2} \hat{\rho}(t)$. The diagonal part is applied on a grid basis set by using the usual fast Fourier transform between the position representation for the potential operator and the impulsion representation for the kinetic operator. The off-diagonal part is treated by a Cayley iteration procedure. $^{52,65}$

\section{OPTIMAL CONTROL THEORY IN DISSIPATIVE SYSTEMS}

We apply the optimal control theory in the density matrix formalism. ${ }^{42,66-68}$ We work in the Liouville space and use the double-space notation, which verifies $\langle\langle A \mid B\rangle\rangle=\operatorname{Tr}\left(A^{\dagger} B\right)$.

The optimal field able to drive the initial density matrix toward the target is determined by variational theory. In reactivity, the functional is usually the probability that the steered density matrix is the target at a given final time $t_{\max }$. The functional is maximized under the constraints that the laser fluence remains acceptable and the Liouville equation is satisfied at any time ${ }^{42,44}$

$$
\begin{aligned}
F[E(t)]= & \left\langle\left\langle\rho_{\mathrm{S}}\left(t_{\max }\right) \mid \rho_{\text {target }}\right\rangle\right\rangle-\int_{0}^{t_{\max }} \mathrm{d} t \alpha(t) \sum_{j} E_{j}^{2}(t) \\
& -\int_{0}^{t_{\max }} \mathrm{d} t\left\langle\left\langle\chi_{\mathrm{S}}(t) \mid\left\{\partial_{t}\left|\rho_{\mathrm{S}}(t)\right\rangle\right\rangle\right.\right. \\
& \left.-i\left(H_{\mathrm{eff}}^{0}-\sum_{j} \mu_{j} E_{j}(t)\right)\left|\rho_{\mathrm{S}}(t)\right\rangle\right\rangle \\
& \left.\left.+\int_{0}^{t} \mathrm{~d} t^{\prime} K\left(t-t^{\prime}\right)\left|\rho_{\mathrm{S}}\left(t^{\prime}\right)\right\rangle\right\rangle\right\}
\end{aligned}
$$

with Lagrange multipliers $\chi_{\mathrm{S}}(t)$ and $\alpha(t)$. The latter is set $\alpha(t)=$ $\alpha_{0} / s(t)$, where $s(t)=\sin ^{2}\left(\pi t / t_{\max }\right)$ is a smooth switching function. Varying the functional leads to three coupled equations: the Liouville equation for $\left.\left|\rho_{\mathrm{S}}(t)\right\rangle\right\rangle$ with an initial condition $\left.\left|\rho_{S}(t=0)\right\rangle\right\rangle$ (usual forward propagation), the Liouville equation for the Lagrange multiplier $\left.\left|\chi_{S}(t)\right\rangle\right\rangle$ which must be solved with a final condition $\left.\left.\left|\chi_{\mathrm{s}}\left(t_{\max }\right)\right\rangle\right\rangle=\left|\rho_{\text {target }}\right\rangle\right\rangle$ so that in practice it is solved by a backward propagation, and the optimum field for each polarization $j$

$$
E_{j}(t)=\left(-s(t) / \alpha_{0}\right) \operatorname{Im}\left(\left\langle\left\langle\chi_{S}\left|\mu_{j}\right| \rho_{S}(t)\right\rangle\right\rangle\right)
$$

The equations are solved by the Rabitz iterative monotonous algorithm. ${ }^{42}$ We have used the improvement proposed in ref 69. At each iteration $k$, the field is given by $E_{j}^{(k)}=E_{j}^{(k-1)}+\Delta E_{j}^{(k)}$, where $\Delta E_{j}^{(k)}$ is calculated by eq 17 .

As mentioned above, each iteration of the OCT scheme consists of two steps: a forward propagation with $\rho_{k}(t=0)=0$ and $\rho_{S}(t=0)$ describing the initial state and a backward propagation with $\chi_{k}\left(t_{\max }\right)=0$ and $\chi_{\mathrm{S}}\left(t_{\max }\right)$ fixed by the target state as the final condition. The equations for the forward propagation are those given in the previous part. A difficulty in the density matrix formalism is to write the equations for the backward propagation. The Liouville equation for the Lagrange multiplier $\chi_{\mathrm{S}}(t)$ reads $^{44}$

$$
\dot{\chi}_{\mathrm{S}}(t)=L_{\mathrm{eff}} \chi_{\mathrm{S}}(t)+\int_{t_{\mathrm{f}}}^{t} \mathrm{~d} t^{\prime} K^{\dagger}\left(t^{\prime}, t\right) \chi_{\mathrm{S}}\left(t^{\prime}\right)
$$

with the memory kernel being

$$
\int_{t_{\mathrm{f}}}^{t} \mathrm{~d} t^{\prime} K^{\dagger}\left(t^{\prime}, t\right) \chi_{\mathrm{S}}\left(t^{\prime}\right)=i \sum_{k}\left[r, \chi_{k}(t)\right]
$$

and

$$
\begin{aligned}
\chi_{k}(t)= & -i \int_{t_{\mathrm{f}}}^{t} \mathrm{~d} t^{\prime} \mathrm{e}^{i \gamma_{k}\left(t^{\prime}-t\right)} T \mathrm{e}^{\int_{t^{\prime}}^{t} L_{\mathrm{S}}\left(t^{\prime \prime}\right) \mathrm{d} t^{\prime \prime}}\left[\alpha_{k} \chi_{\mathrm{S}}\left(t^{\prime}\right) r\right. \\
& \left.-\tilde{\alpha}_{k} r \chi_{\mathrm{S}}\left(t^{\prime}\right)\right]
\end{aligned}
$$

This leads to the following system of coupled equations:

$$
\begin{aligned}
& \dot{\chi}_{\mathrm{S}}(t)=L_{\mathrm{eff}} \chi_{\mathrm{S}}(t)+i \sum_{k}\left[r, \chi_{k}(t)\right] \\
& \dot{\chi}_{k}(t)=-\left(i \gamma_{k}-L_{\mathrm{S}}\right) \chi_{k}(t)-i\left[\alpha_{k} \chi_{\mathrm{S}}(t) r-\tilde{\alpha}_{k} r \chi_{\mathrm{S}}(t)\right]
\end{aligned}
$$

The procedure used to solve this system is the same as that for the forward propagation.

\section{RESULTS}

To fully assess the role of dissipation within the context of laser controlled isomerization considered in this work, we proceed in several steps, as shown below. The control objective is to actively drive the isomerization reaction from the right potential well (configuration a in Figure 1) to the left potential well (configuration c, see Figure 1). In terms of the eigenstates of the one-dimensional model employed, this results in a controlled transition from the initial $\phi_{\nu=0}$ state to the target state $\phi_{\nu=4}$. As shown in Figure 2, the $\nu=4$ eigenstate is entirely localized in the product well. At the temperature of $300 \mathrm{~K}$ we consider in this work, the initial state is almost a pure state, since almost the entire population is in the $\nu=0$ state at thermal equilibrium. All the examples are given for a bath spectral density with a cutoff $\omega_{\mathrm{c}}=1700 \mathrm{~cm}^{-1}$. The cutoff is chosen so that the active frequencies lying in the range 1000$2000 \mathrm{~cm}^{-1}$ interact with the bath. Similar results have been obtained with $\omega_{c}=900$ and $400 \mathrm{~cm}^{-1}$. However, for these values, the coupling is expected to be weaker. The simulations were done with five Matsubara matrices, a time step $\mathrm{d} t=0.19$ fs, and an equidistant grid with 64 points for the potential and the dipole moment. The application of the kinetic operator was performed using the Fourier method, ${ }^{70}$ which in our case supports energies up to $1.4 \mathrm{eV}$, which is enough for the processes described below.

Optimal Control without Dissipation. We optimize a field with a duration as short as possible taking into account a limiting value of the field amplitude. This is important, since it may in a real experiment lead to strong field effects like multiphoton ionization or effect of polarization. Specifically, we use a maximum field value of $0.02 \mathrm{au}\left(1.028 \times 10^{8} \mathrm{~V} \mathrm{~cm}^{-1}\right.$ and in some cases a value of 0.03 au for analysis). A rough estimate based on the reactant and product structures reveals that larger field values may lead to non-negligible polarization effects. We use two linear polarizations along the directions $x$ and $y$ after an analysis of the dipole matrix elements. The molecules are assumed to be oriented in the laboratory. In this first step of control without dissipation, we choose the trial field in such a 
way that the localized vibrational states in the initial well up to the middle of the barrier are early populated in order to initiate the vibrational excitation in the reactant well. The trial field is given by $E_{j}^{(0)}=\varepsilon_{0} s(t) \sum_{k=1}^{n_{j}} \cos \left(\omega_{k} t\right)$ with $j=x, y$. We choose three frequencies for the polarization $x, \omega_{12}\left(1027.0 \mathrm{~cm}^{-1}\right), \omega_{23}$ $\left(1004.6 \mathrm{~cm}^{-1}\right)$, and $\omega_{34}\left(977.7 \mathrm{~cm}^{-1}\right)$, and two for $y, \omega_{46}(943.2$ $\left.\mathrm{cm}^{-1}\right)$ and $\omega_{68}\left(892.0 \mathrm{~cm}^{-1}\right)$, with an amplitude of $0.01 \mathrm{au}$ $\left(5.142 \times 10^{7} \mathrm{~V} \mathrm{~cm}^{-1}\right)$. The probability to be in the target state at the end of the pulse converges at $99.9 \%$ after about 200 iterations. However, to force a limit maximum field amplitude, we divide the amplitude by a factor of 2 and let it iterate again for 100 iterations. This process is repeated twice to obtain the desired control field $E_{x / y}^{(1)}$, which will be referred to as "field 1".

In order to analyze the results of the dissipation-free control, we calculate the populations of different levels or groups of levels. To this end, and for later reference, we define groups of levels through $S_{\mathrm{r}}=\{1,2,3,5,7,9,11,13,16,18,20,22\}$, which are all states localized (or preferentially localized) in the right potential well (without the reactant level, $\nu=0$ ), $S_{1}=$ $\{6,8,10,12,14,15,17,19,21,23\}$, which are states localized preferentially in the left well (excluding the target state $\nu=4$ ), and $S_{\text {del }}=\{\nu: \nu \geq 24\}$, which comprises all states lying energetically above the barrier. These states are delocalized across the barrier and have a large amplitude in both wells. Using these definitions, we can define projectors onto individual levels or subspaces by

$$
\hat{P}_{\nu}=\left|\phi_{\nu}\right\rangle\left\langle\phi_{\nu}\left|, \quad \hat{P}_{1, \mathrm{r}, \mathrm{del}}=\sum_{\nu \in S_{1, \mathrm{r}, \mathrm{del}}}\right| \phi_{\nu}\right\rangle\left\langle\phi_{\nu}\right|
$$

which are used within the density matrix approach to calculate the populations

$$
P_{q}^{(\kappa)}=\operatorname{tr}\left(\rho_{\mathrm{S}} \hat{P}_{q}\right)
$$

where we use the superscript $(\kappa)$ to identify the results obtained with different control fields $E_{x / y}^{(\kappa)}$, as detailed below, and $q$ stands for the different subspaces considered, i.e., $q=\nu, \mathrm{l}, \mathrm{r}, \mathrm{del}$. Furthermore, we will use the population of the initial state and the target state, which in our case are simply $P_{i}^{(\kappa)}=P_{0}^{(\kappa)}$ and $P_{t}^{(\kappa)}$ $=P_{4}^{(\kappa)}$. In Figure 3 , we show $P_{i}^{(1)}, P_{t}^{(1)}, P_{\mathrm{r}}^{(1)}, P_{1}^{(1)}$, and $P_{\mathrm{del}}^{(1)}$, obtained using the field $E_{x / y}^{(1)}$, as a function of time. During the

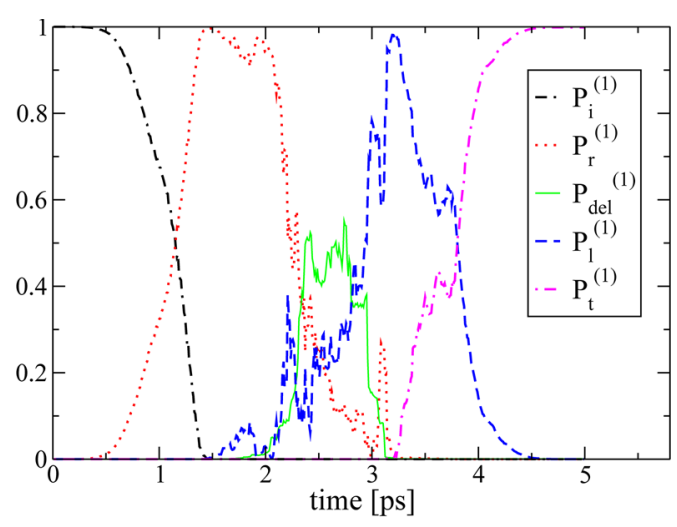

Figure 3. Evolution of groups of levels, specifically the ground state $\nu$ $=0$, localized in the reactant well, the target state $\nu=4$, which is the lowest state localized in the product well, $\nu \in S_{\mathrm{r} / \mathrm{l}}$, with $S_{\mathrm{r} / 1}$ regrouping all levels localized in the left or right potential well, and $\nu \in S_{\text {del }}$ with $S_{\text {del }}$ being all levels with $\nu \geq 24$, which are above the barrier and delocalized from reactant to product. The cutoff is $1700 \mathrm{~cm}^{-1}$ and $\lambda=$ 0.006 au. first picosecond, the initial state is almost entirely depopulated, due to the heating in the right potential well, indicated by the rise in $P_{\mathrm{r}}^{(1)}$. Then, between 2 and 3 ps, almost $50 \%$ of the population is in the delocalized states. Then, toward the end of the pulse, the system is driven into the left well, shown by the maximum of $P_{1}^{(1)}$, and eventually cooled down and the population is accumulated in the target state, $P_{t}^{(1)}$. In this dissipation-free scenario, we see that the control achieves almost $100 \%$ of population transfer to the target state within the time window of 5 ps. This result is the starting point for the control including dissipation.

Optimal Control with Dissipation. To assess the influence of dissipation onto the isomerization and to answer the question as to which extent the control can compensate for the effects of the environment, we proceed in two steps: first, we analyze the isomerization dynamics including dissipation but driving the system with the control field $E_{x / y}^{(1)}$ obtained without dissipation. In a second step, we calculate a new control field, now including the dissipation already at the design stage, i.e., using the methodology presented in the previous chapter. This field will be denoted by $E_{x / y}^{(2)}$.

Since it is known that the results of an OCT calculation depend on the initial guess, we have also optimized the field within the dissipative OCT approach using the result of the field-free optimization, $E_{x / y}^{(1)}$, as a starting point for the dissipative OCT iterations. However, this procedure has given fields with slightly lower target success, and consequently, the results in this paper are obtained using $E_{x / y}^{(0)}$ as an initial guess. In Figure 4, we show the objective, i.e., the final population $P_{t}^{(\kappa)}$ of the target state for the pulse obtained without dissipation, $P_{t}^{(1)}$, and the result of our dissipative control methodology, $P_{t}^{(2)}$. As expected, we find that the objective decreases with increasing coupling strength. For the

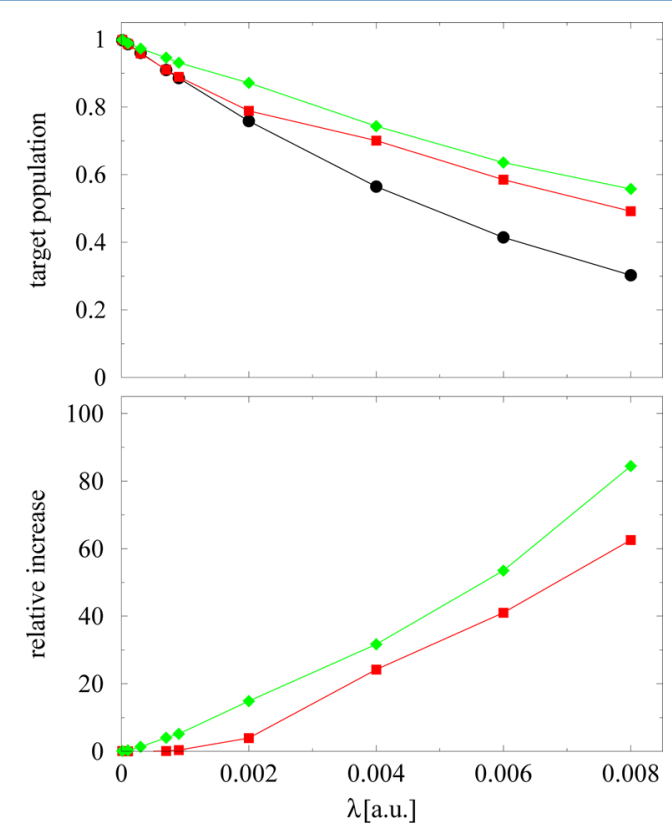

Figure 4. Upper panel: Final target state population using different control pulses: $P_{t}^{(1)}$ (black, circles) using a field obtained without dissipation, $P_{t}^{(2)}$ using a field obtained by the methodology presented, which includes dissipation in the design stage, for different field thresholds (red, squares: $E_{\max }=0.02$ au; green, diamonds: $E_{\max }=0.03$ au). Lower panel: relative increase, $\left(P_{t}^{(2)}-P_{t}^{(1)}\right) / P_{t}^{(1)}$, field thresholds as above. The cutoff frequency is $1700 \mathrm{~cm}^{-1}$. 
range of $\lambda$ chosen, the decrease in the case where the dissipative-free optimized field was used is drastic, falling as low as $40 \%$, as compared to the $100 \%$ efficiency of the same pulse under dissipative-free conditions. Already, this finding shows the drastic effect the bath has on the isomerization dynamics. Regarding $P_{t}^{(2)}$, one clearly sees that including the dissipation at the design stage of the control pulse modifies the field in such a way that, for a given value of the system-bath coupling $\lambda$, the objective is significantly increased, as compared to the result obtained when using the field optimized without dissipation. As indicated in the Optimal Control Theory in Dissipative Systems section, we have chosen a maximal field strength of the electric fields obtained. This threshold value is set to $E_{\max }=$ $0.02 \mathrm{au}$ in most of the work presented. In Figure 4, we have also included results based on a threshold value of $E_{\max }=0.03 \mathrm{au}$, to analyze the dependence of our results on its value. In the lower panel, this increase is given as the ratio with respect to the final state population, $\left(P_{t}^{(2)}-P_{t}^{(1)}\right) / P_{t}^{(1)}$, and we find an important increase up to $63 \%$ (for $E_{\max }=0.02 \mathrm{au}$ ) or even $80 \%$ (for $E_{\max }=$ $0.03 \mathrm{au}$ ). Not surprising, we find that allowing for higher field values leads to an even better result.

As one of the main results of this paper, we see that OCT, combined with the auxiliary density matrix approach to account for dissipation at the design stage, does indeed manage to improve on the dissipative-free control fields. It finds modified fields, which lead to much higher final state populations. The proposed methodology has thus proven to be able to react onto the dissipation and create fields which can compensate for dissipation effects.

In what follows, we want to analyze the isomerization dynamics in an exemplary way for one value of $\lambda$, namely, $\lambda=$ $0.006 \mathrm{au}$, in order to get a physical picture of how the control manages to fight dissipation.

\section{PHYSICAL PICTURE}

To analyze the effect of dissipation on the nuclear dynamics and to elucidate the strategy found by the control when including dissipation, we will compare in this section the OCT pulses obtained with and without including the dissipation, and the resulting dynamics, which in both cases includes the thermal bath.

We start with comparing the pulses obtained with and without the influence of the bath. In Figure 5, left panel a shows the two components of the electric field $E_{x}^{(1)}$ and $E_{y}^{(1)}$ as a spectrogram, obtained by a Gabor transform, ${ }^{70}$ as detailed in ref 47. In the right panel $b$, we show the corresponding field components when using eqs 13,17 , and 21 . As expected, the fields show distinct differences, especially around 2 ps, at the center of the pulse. At the beginning, both pulses start with frequencies in the range of $600-1000 \mathrm{~cm}^{-1}$. These frequencies correspond to the spacings between the levels $\nu \in S_{r}$ located in the reactant potential well (the right well in Figure 2) and lead to vibrational ladder climbing in the reactant well. This is especially driven by $E_{x}$, since $\mu_{x}$ shows a very strong variation in the reactant well region. Then, around 2 ps, in both cases strong $E_{y}$ components appear. At that time, the system is close to the transition state, where $\mu_{x}$ shows a minimum and is rather flat, while $\mu_{y}$ has a very strong $r$-dependence. When comparing the fields obtained without dissipation (panels a in Figure 5) to the ones obtained including dissipation (panels b in Figure 5), one sees clear differences both in the $x$ and $y$ components. Specifically, the field obtained by including the dissipation produces spectral components at energies higher than 1600

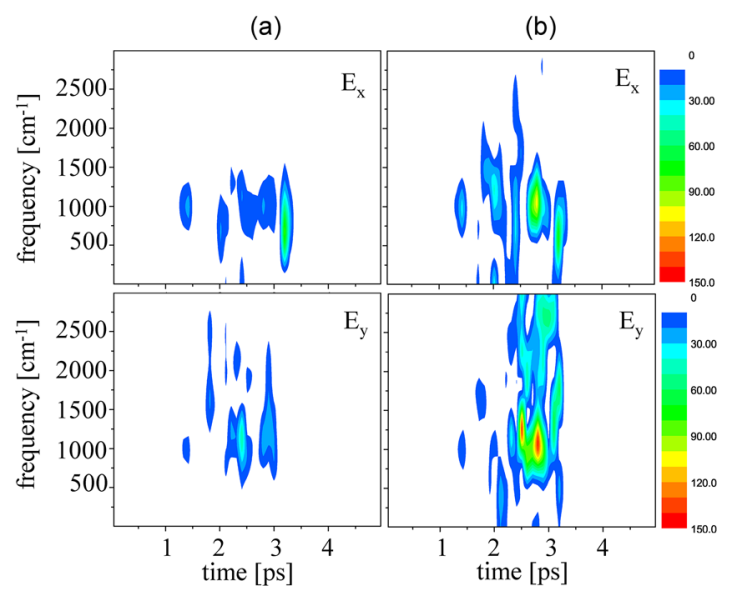

Figure 5. Spectrograms of the control pulses considered, when applying OCT without dissipation (a) or including dissipation at the design stage (b), using the presented formalism, with $\omega_{\mathrm{c}}=1700 \mathrm{~cm}^{-1}$, $\lambda=0.006 \mathrm{au}$, and $E_{\max }=0.02 \mathrm{au}$. The intensity scale, in arbitrary units, is the same for all cases.

$\mathrm{cm}^{-1}$. To analyze the origin of these components, we show in Figure 6 the populations of high-lying levels in the reactant well and the lowest delocalized ones. Specifically, we plot the levels $\nu \in S_{r}, \nu>13$, and $\nu \in S_{\mathrm{del}}$.

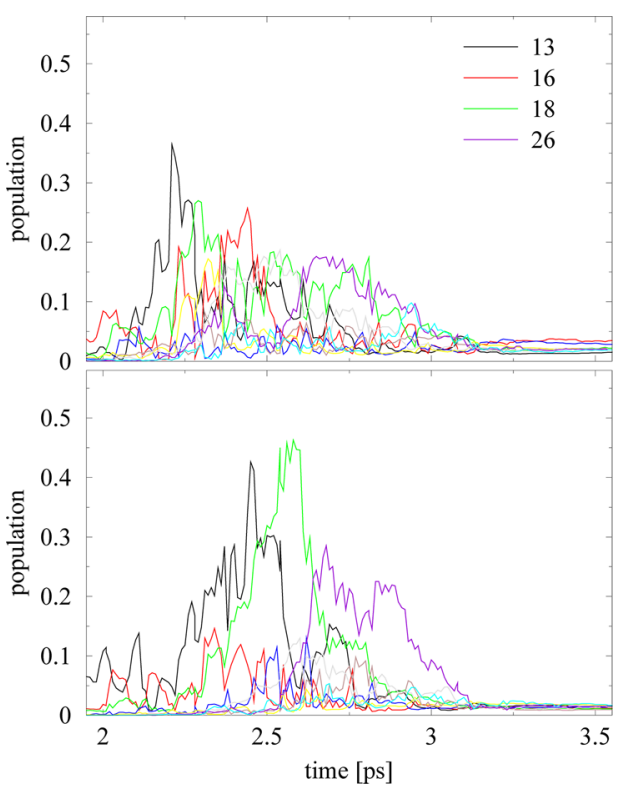

Figure 6. Populations of different levels during the barrier crossing. Upper panel: $P_{v}^{(1)}(\nu$ as indicated) using the field obtained without dissipation. Lower panel: $P_{v}^{(2)}$ using the field obtained with dissipation. The conditions are the same as those in Figure 5.

When comparing the time-dependent populations depicted in Figure 6 to the spectrogram presented in Figure 5, note the difference in scale: Figure 6 concentrates on the barrier crossing, which occurs between 2 and 3 ps. The field components prior to 2 ps lead to vibrational excitations in the reactant well and are not shown in Figure 6. When looking at the quantum dynamics based on the pulse obtained without dissipation (top panel), one sees a rather broad distribution of all levels shown. However, when the pulse obtained with dissipation is used for the quantum propagation (lower panel), only a few states are populated preferentially. Inspection of the 
populations in the different levels reveals that, in this latter case, after the excitation with the reactant well, the passage across the barrier is mainly performed via the states $\nu=13 \rightarrow \nu=18 \rightarrow \nu$ $=26$ (see Figure 6, lower panel). In particular, the direct excitation $\nu=13 \rightarrow \nu=18$ explains the high frequency component, since the energy difference of these two states is approximately $1600 \mathrm{~cm}^{-1}$.

This scenario, however, requires fields of higher intensity. These rather high intensities lead to extensive absorption and stimulated emission processes between the quantum levels, as can be seen from Figure 6. This multilevel Rabi flopping also means that the field interaction is clearly nonperturbative. As a consequence, there is no simple relationship between the pulse intensity and the energy transferred to the system. At the end of the isomerization process, the system is primordially in the state $\nu=5$, while, during the laser interaction, highly excited levels are transiently populated. However, nearly the same excited states are involved with and without dissipation and only the mechanism is different. The average energy $\left\langle H_{\text {eff }}^{0}\right\rangle$ reaches quasi the same maximum value even if the intensity of the field fighting dissipation is higher.

To summarize, we see that, in the case that the dissipation is included in the OCT iterations, the barrier crossing is effectuated in a more rapid way, preferentially involving fewer states.

To show the accelerated barrier crossing obtained when including dissipation, we plot in Figure 7 the total population of

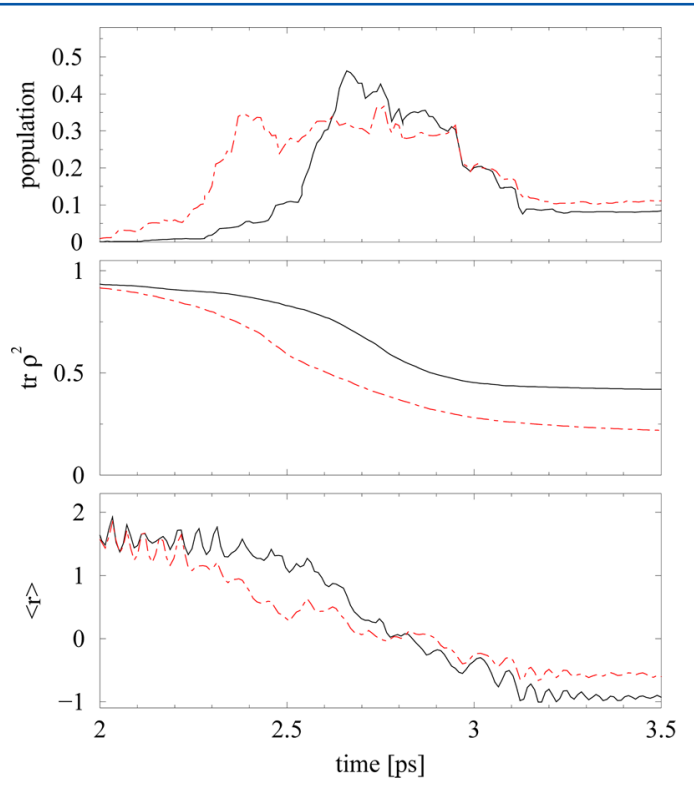

Figure 7. Comparison of dissipative barrier crossing using control fields that include dissipation or not. Upper panel: population in the delocalized states $P_{\mathrm{del}}^{(1)}\left(\right.$ red), $P_{\mathrm{del}}^{(2)}$ (black). Middle panel: $\operatorname{tr}\left(\rho_{\mathrm{S}}^{2}\right)$ for the corresponding density matrices. Bottom panel: position expectation value. Full lines, $E^{(2)}$ field; dashed lines, $E^{(1)}$ field. The conditions are the same as in Figure 5.

all delocalized states as defined in the preceding section (upper panel). When comparing the time evolution of this population, one sees that for the control field $E_{x / y}^{(1)}$ (obtained without dissipation) the system spends 800 fs (value taken at $P_{\text {del }}=$ 0.25 , which is approximately the full width at half-maximum) in these states, whereas in the dynamics driven by $E_{\mathrm{del}}^{(2)}$, obtained with dissipation at the design stage, this time is significantly reduced to about $400 \mathrm{fs}$.
The quantum dynamics of the isomerization process leads to decoherence due to the coupling to the environment. This can be quantified by monitoring $\operatorname{tr} \rho_{\mathrm{S}}{ }^{2}$, which is a measure of the purity of the density matrix. This quantity is bounded by 0 and 1 , a value of 1 corresponding to a pure state. ${ }^{71}$ It can be shown that a decrease in $\operatorname{tr} \rho_{\mathrm{S}}{ }^{2}$ corresponds to a decay of the offdiagonal elements of the density matrix when represented in a basis of eigenfunctions of the system Hamiltonian. ${ }^{71}$ However, considering $\operatorname{tr} \rho_{\mathrm{S}}^{2}$ directly has the advantage of being independent of any representation, and analyzing its temporal evolution while the system undergoes isomerization allows one to directly relate the pulse-induced dynamics with decoherence. To this end, we trace in Figure 7 this quantity together with the population of the delocalized states, $P_{\mathrm{del}}^{(1 / 2)}$, and the position expectation value.

Comparing the upper panel to the lower panel, one clearly sees that the decoherence, i.e., the loss of purity, coincides with the population in the delocalized states. These states describe large amplitude motion, ranging from $r=-2 \AA$ to $r=2 \AA$, and can thus efficiently couple to the bath via the coupling operator $r$.

A second observation is that the higher target state population is obtained with a density matrix of higher purity. The result clearly confirms that decoherence is the key process opposing coherent control, as the juxtaposition of the word "coherent" already indicates. If control within a dissipative environment wants to be efficient, it has to create fields that drive the system in such a way as to minimize decoherence. In our system, the strategy found by the proposed method combining OCT with the auxiliary density matrix method is clearly visible from Figure 7: the control including dissipation has created a field that drives the system in such a way that it spends less time in the delocalized states, where the loss of coherence is preponderant.

This accelerated barrier crossing leads to a reduced loss in purity, and to a higher yield in the control objective. This physical picture can also be seen when plotting the position expectation value as a function of time for the above-mentioned cases: in Figure 7, lower panel, one clearly sees the more rapid isomerization process, when the bath is included at the design stage. Having established this very simple but interesting and efficient mechanism found by the OCT method including dissipation, we now turn to the $\lambda$-dependence and analyze to what extent this accelerated barrier crossing evolves with increasing system-bath coupling. To this end, we show in Figure $8 P_{\mathrm{del}}^{(\kappa)}$ as a function of time, for different values of $\lambda$. In the upper panel, the fields were constrained to be lower than $0.02 \mathrm{au}$, whereas, in the lower panel, this threshold value was chosen to be 0.03 au.

In the upper panel, one clearly sees that, as a function of $\lambda$, the crossing time decreases until it reaches about $400 \mathrm{fs}$, where it settles. The leveling for large values of $\lambda$ is due to the field constraints: to make the objective increase, the control opts for an accelerated passage across the delocalized states but at the price of a higher intensity of the pulses. Once the OCT iterations reach this limiting maximal intensity, the control is not pursued any further but stopped at this iteration stage. In the lower panel, where a higher limit was chosen for $E_{\max }$ this decreasing of the barrier crossing time levels at a lower value of $\lambda$. This behavior also explains that accepting a higher value of the maximal field strength allows for pulses that are more efficient in their aim to create control of nuclear dynamics in dissipative environments, as clearly visible in Figure 8. 

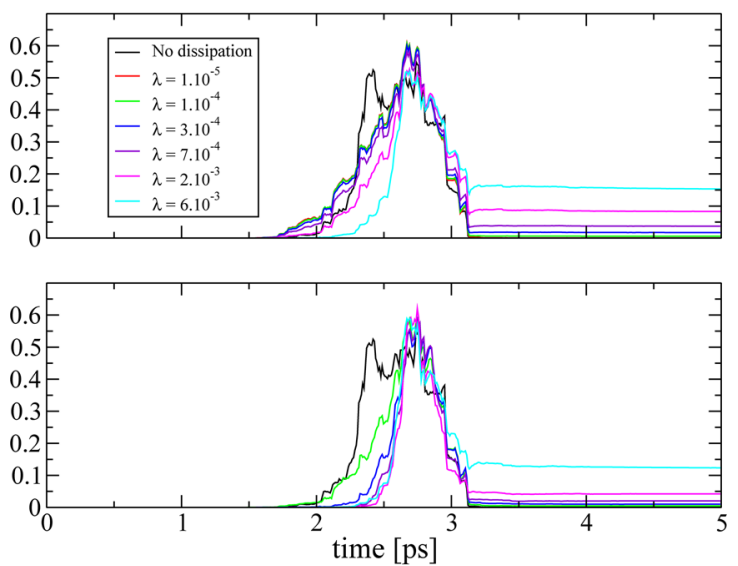

Figure 8. Population in the delocalized states $P_{\mathrm{del}}^{(2)}$ for different system-bath couplings $\lambda$ (in atomic units). Upper panel: field threshold of $E_{\max }=0.02$ au. Bottom panel: field threshold of $E_{\max }=$ $0.03 \mathrm{au}$. Clearly to be seen is the shortening of the barrier crossing time, as compared to the dissipation-free case.

\section{CONCLUSION}

In this work, we have presented theoretical studies of optimal control of an isomerization process including dissipative effects due to a model environment assumed to be described by an Ohmic spectral density. The example chosen is the Cope rearrangement of the predominant species formed by the dimerization of methyl-cyclopentadienylcarboxylate. This dimerization leads to Thiele's ester, which, via the Cope transition state, can isomerize to another stable form of higher energy. On the basis of a one-dimensional reaction path model, the isomerization was investigated with a dissipative system-bath model. The interaction with the bath is treated within a nonMarkovian master equation approach, using the auxiliary density matrix approach first proposed in ref 52. This approach has the advantage of being local in time, while taking memory and the effects of external driving fields fully into account. We show that this quantum dynamical approach can be combined with OCT, and the developed formalism can be used to construct control fields in the presence of dissipation.

For the example chosen with different frequency cutoff, we always found that including dissipation leads to different control fields, as compared to those obtained by dissipative-free OCT, and to higher target yields. Pictorally speaking, including dissipation at the design stage enables the control algorithm to react on the environment-induced decoherence and to change the field-induced dynamics in order to obtain a better control result. The work is based on a realistic reaction path model where the potential energy surface as well as the dipole moments are obtained by electronic structure calculations. Furthermore, the fields are constrained to avoid unphysical field strengths. Our results thus show, despite being a onedimensional model, that the proposed system could be a good candidate to experimentally demonstrate isomerization control in a complex environment. Even though our fields are not predictive due to the limitations of the model, the results nevertheless show that a high degree of controllability should be possible. Extension of the model, to yield a more realistic description, can be achieved by considering a second degree of freedom to describe the isomerization, as detailed in ref 47 . While the methodology presented in this work can in a straightforward way be applied for systems with more dimensions, the numerical feasibility remains to be seen in future work, which is underway. The power of the auxiliary density matrix method approach coupled with optimal control theory will be confirmed when dealing with more realistic spectral densities coming from experience or from dynamical simulations. This topic is also in progress.

\section{AUTHOR INFORMATION}

\section{Corresponding Author}

*E-mail: chris@irsamc.ups-tlse.fr (C.M.); michele.desouterlecomte@u-psud.fr (M.D.-L.).

\section{Notes}

The authors declare no competing financial interest.

\section{ACKNOWLEDGMENTS}

We would like to thank M. Ndong for providing starting fields, and also valuable insights in various aspects of OCT. Financial support through the ANR "PROCONTROL" is gratefully acknowledged. Dr. G. Dive is a research fellow of the F.R.S.FNRS of Belgium.

\section{REFERENCES}

(1) Tannor, D.; Rice, S. A. J. Chem. Phys. 1985, 83, 5013.

(2) Pierce, A. P.; Dahleh, M. A.; Rabitz, H. Phys. Rev. A 1987, 4950.

(3) Shi, S.; Rabitz, H. J. Chem. Phys. 1989, 364.

(4) Kosloff, R.; Rrice, S.; Gaspard, P.; Tersigny, S.; Tannor, D. Chem. Phys. 1989, 139, 201.

(5) Brumer, P.; Shapiro, M. Annu. Rev. Phys. Chem. 1992, 4, 257.

(6) Warren, W.; Rabitz, H.; Dahleh, M. Science 1993, 259, 1581.

(7) Dantus, M.; Lozokov, V. V. Chem. Rev. 2004, 104, 1813.

(8) Manz, J. Femtochemistry and femtobiology: Ultrafast Reaction Dynamics at atomic-scale resolution; Imperial College Press: London, 1997.

(9) Manz, J., Wöste, L., Eds. Femtosecond Chemistry; Wiley-VCH, Weinheim, Germany, 1995.

(10) Barth, I.; Manz, J.; Shigeta, Y.; Yagi, K. J. Am. Chem. Soc. 2006, $128,7043-7049$.

(11) Znakovskaya, I.; von de Hoff, P.; Zherebtsov, S.; Wirth, A.; Herrwerth, O.; Vrakking, M.; de Vivie-Riedle, R; Kling, M. F. Phys. Rev. Lett. 2009, 103, 103002.

(12) Klamroth, T. J. Chem. Phys. 2006, 124, 144310.

(13) Ulusoy, I. S.; Nest, M. J. Am. Chem. Soc. 2011, 133, 2023020236.

(14) Korolkov, M. V.; Manz, J.; Paramonov, G. K. J. Chem. Phys. 1996, 105, 10874.

(15) Doslic, N.; Sundermann, K.; Gonzalez, L.; Mo, O.; GiraudGirard, J.; Kühn, O. Phys. Chem. Chem. Phys. 1999, 1, 1247.

(16) Kühn, O. J. Phys. Chem. A 2002, 106, 7671.

(17) Hoki, K.; Ohtsuki, Y.; Fujimura, Y. J. Chem. Phys. 2001, 114, 1575-1581.

(18) Vogt, G.; Krampert, G.; Niklaus, P.; Nuemberger, P.; Gerber, G. Phys. Rev. Lett. 2005, 94, 068305.

(19) Hoki, K.; Brumer, P. Phys. Rev. Lett. 2005, 95, 168305.

(20) Vogt, G.; Nuernberger, P.; Brixner, T.; Gerber, G. Chem. Phys. Lett. 2006, 433, 433.

(21) Kotur, M.; Weinacht, T.; Pearson, B. J.; Matsika, S. J. Chem. Phys. 2009, 130, 134311.

(22) Mitrić, R.; Petersen, J.; Bonačić Koutecký, V. Phys. Rev. A 2009, $79,053416$.

(23) Prokhorenko, V. I.; Halpin, A.; Johnson, P. J. M.; Miller, R. J. D.; Brown, L. S. J. Chem. Phys. 2011, 134, 085105.

(24) Shah, S. P.; Rice, S. A. J. Chem. Phys. 2000, 113, 6536-6541.

(25) Gong, J.; Ma, A.; Rice, S. J. Chem. Phys. 2005, 122, 204505.

(26) Sugny, D.; Kontz, C.; Ndong, M.; Justum, Y.; Dive, G.; Desouter-Lecomte, M. Phys. Rev. A 2006, 74, 043419.

(27) Gräfe, S.; Meier, C.; Engel, V. J. Chem. Phys. 2005, 122, 184103. 
(28) Cheng, T.; Darmawan, H.; Brown, A. Phys. Rev. A 2007, 75, 013411.

(29) Kondorskiy, A.; Nakamura, H. Phys. Rev. A 2008, 77, 043407.

(30) Kurosaki, Y.; Artamonov, M.; Ho, T.-S.; Rabitz, H. J. Chem. Phys. 2009, 131, 044306.

(31) Gonzalez, L.; Kroner, D.; Sola, I. J. Chem. Phys. 2001, 115, 2519.

(32) Artamonov, M.; Ho, T.-S.; Rabitz, H. Chem. Phys. 2008, 328, 147-155.

(33) Artamonov, M.; Ho, T.-S.; Rabitz, H. J. Chem. Phys. 2006, 124, 064306.

(34) Zhang, M.; Gong, J.; Ma, A.; Rice, S. J. Chem. Phys. 2007, 127, 144501.

(35) Brif, C.; Chakrabarti, R.; Rabitz, H. New J. Phys. 2010, 12, 075008.

(36) Naundorf, H.; Sundermann, K.; Kühn, O. Chem. Phys. 1999, $240,163$.

(37) Kuhl, A.; Domcke, W. Chem. Phys. 2000, 259, 227.

(38) Kühn, O.; Naundorf, H. Phys. Chem. Chem. Phys. 2003, 5, 79.

(39) Bruggemann, B.; Pullerits, T.; May, V. J. Photochem. Photobiol. A 2007, 190, 372.

(40) Jirari, H.; Potz, W. Phys. Rev. A 2005, 72, 013409.

(41) Beyvers, S.; Ohtsuki, Y.; Saalfrank, P. J. Chem. Phys. 2006, 124, 234706.

(42) Zhu, W.; Rabitz, H. J. Chem. Phys. 2003, 118, 6751-6757.

(43) Shuang, F.; Rabitz, H. J. Chem. Phys. 2006, 124, 154105.

(44) Ohtsuki, Y. J. Chem. Phys. 2003, 119, 661-671.

(45) Xu, R.; Yan, Y.; Ohtsuki, Y.; Fujimura, Y.; Rabitz, H. J. Chem. Phys. 2004, 120, 6600-6608.

(46) Sugny, D.; Ndong, M.; Lauvergnat, D.; Justum, Y.; DesouterLecomte, M. J. Photochem. Photobiol., A 2007, 190, 359.

(47) Dive, G.; Robiette, R.; Chenel, A.; Ndong, M.; Meier, C.; Desouter-Lecomte, M. Theor. Chem. Acc. 2012, 131, 1236.

(48) Thiele, J. Chem. Ber. 1901, 34, 68.

(49) Marchand, A.; N. T., V. V.; Zhao, D. Tetrahedron 1993, 49, $2613-2620$

(50) Westermann, T.; Brodbeck, R; Rozhenko, A. B.; Schoeller, W.; Manthe, U. J. Chem. Phys. 2011, 135, 184102.

(51) Yan, Y.; Xu, R. Annu. Rev. Phys. Chem. 2005, 56, 187-219.

(52) Meier, C.; Tannor, D. J. J. Chem. Phys. 1999, 111, 3365-3376.

(53) Kleinekathöfer, U. J. Chem. Phys. 2004, 121, 2505-2514.

(54) Pomyalov, A.; Meier, C.; Tannor, D. J. Chem. Phys. 2010, 370, 98

(55) Becke, A. D. J. Chem. Phys. 1993, 98, 5648-5652.

(56) Frisch, M. J.; et al. Gaussian 09, revision A.1; Gaussian Inc.: Wallingford, CT, 2009.

(57) Francl, M. M.; Pietro, W. J.; Hehre, W. J.; Binkley, J. S.; Gordon, M. S.; DeFrees, D. J.; Pople, J. A. J. Chem. Phys. 1982, 77, 3654-3665.

(58) Garg, A.; Onuchic, J. N.; Ambegaokar, V. J. Chem. Phys. 1985, $83,4491-4503$.

(59) Leggett, A. J.; Chakravarty, S.; Dorsey, A. T.; Fisher, M. P. A.; Garg, A.; Zwerger, W. Rev. Mod. Phys. 1987, 59, 1-85.

(60) Breuer, H.-P.; Petruccione, F. The theory of open quantum systems; Wiley: New York, 2002.

(61) May, V.; Kühn, O. Charge and Energy Transfer Dynamics in Molecular Systems; Wiley-VCH: Berlin, Germany, 2011.

(62) Tanaka, M.; Tanimura, Y. J. Chem. Phys. 2010, 132, 214502.

(63) Pomyalov, A.; Tannor, D. J. Chem. Phys. 2010, 123, 204111.

(64) Feit, M. D.; Fleck, J. A.; Steiger, A. J. Comput. Phys. 1982, 47, 412-433.

(65) Press, W.; Flannery, B.; Teukolsky, S.; Vetterling, W. Numerical Recipes; Cambridge University Press: Cambridge, U.K., 1989.

(66) Ohtsuki, Y.; Zhu, W.; Rabitz, H. J. Chem. Phys. 1999, 110, 9825-9832.

(67) Ohtsuki, Y.; Turinici, G.; Rabitz, H. J. Chem. Phys. 2004, 120, 5509.

(68) Ohtsuki, Y.; Teranishi, Y.; Saalfrank, P.; Turinici, G.; Rabitz, H. Phys. Rev. A 2007, 75, 033407.

(69) Palao, J. P.; Kosloff, R. Phys. Rev. Lett. 2002, 89, 188301.
(70) Sugawara, M.; Fujimura, Y. J. Chem. Phys. 1994, 100, 56465655.

(71) Tannor, D. J. Quantum Mechanics: A Time Dependent Perspective; University Science Book: Sausalito, CA, 2007. 